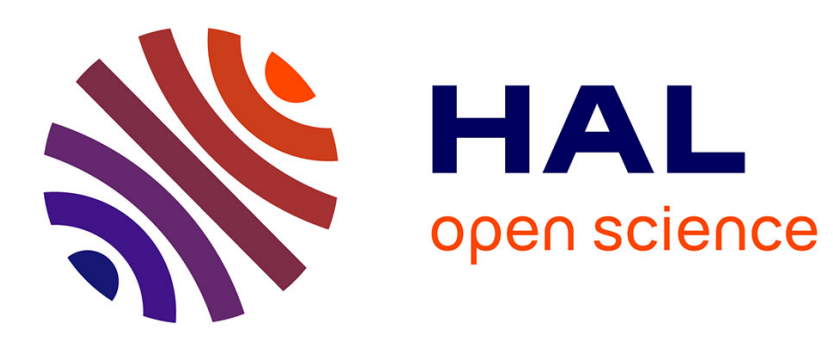

\title{
A simple method to compute standard two-fluid models
} Jean-Marc Hérard, Olivier Hurisse

\section{To cite this version:}

Jean-Marc Hérard, Olivier Hurisse. A simple method to compute standard two-fluid models. International Journal of Computational Fluid Dynamics, 2005, 19 (7), pp.475-482. 10.1080/10618560600566885 . hal-01293567

\section{HAL Id: hal-01293567 \\ https://hal.science/hal-01293567}

Submitted on 25 Mar 2016

HAL is a multi-disciplinary open access archive for the deposit and dissemination of scientific research documents, whether they are published or not. The documents may come from teaching and research institutions in France or abroad, or from public or private research centers.
L'archive ouverte pluridisciplinaire HAL, est destinée au dépôt et à la diffusion de documents scientifiques de niveau recherche, publiés ou non, émanant des établissements d'enseignement et de recherche français ou étrangers, des laboratoires publics ou privés. 


\title{
A SIMPLE METHOD TO COMPUTE STANDARD TWO-FLUID MODELS
}

\author{
Jean-Marc Hérard ${ }^{1,2}$, Olivier Hurisse ${ }^{1,2}$ \\ ${ }^{1}$ Électricité de France, Division Recherche et Développement, \\ Département Mécanique des Fluides et Transferts Thermiques, \\ 6 quai Watier, 78401 Chatou cedex, FRANCE \\ jean-marc.herard@edf.fr, olivier.hurisse@edf.fr \\ Phone : (33) 1308770 37, fax : 33) 130877916 \\ ${ }^{2}$ Université de Provence, Centre de Mathématiques et d'Informatique, \\ Laboratoire d'Analyse, Topologie et Probabilités - UMR 6632, \\ 39 rue Joliot Curie, 13453 Marseille cedex 13, FRANCE
}

\begin{abstract}
We describe in this paper a tool to compute approximate solutions of standard two-fluid models with an equilibrium pressure assumption. The basic approach takes its grounds in the two-fluid two-pressure formalism, and takes advantage of the relaxation techniques. The method may be used to compute either the single pressure or the two-pressure model, depending on the size of mesh which is used. It is also shown on the basis of a simple numerical experiment that the local equilibrium assumption may lead to a blow-up of the numerical solution on fine meshes, even if one accounts for drag stabilizing effects .
\end{abstract}

Keywords: Two-phase flows / Two-fluid models / Relaxation method / Finite Volumes / Approximate Riemann solvers /

\section{Introduction}

There has been a wide debate over the past thirty years in the two-phase flow community, which concerns the potential loss of hyperbolicity of standard two-fluid models, and its consequences in terms of industrial predictions of two-phase flows. A first idea that emerged a long time ago is the following: even if the standard two-fluid model may lead in some test cases to an ill-posed initial value problem, this will never have negative consequences since one uses rather coarse meshes and meanwhile the upwinding flavour will guarantee enough stability to compensate emerging inner instability of the set of PDE. Actually, this might make sense provided that we deal with discrete 
models, and not with continuous models. A second point which is also emphasised is that some (expected small) interfacial transfer terms in the momentum equation (namely $\beta \frac{\partial \alpha}{\partial x}$ ) may become predominant in "dangerous" situations. This was first pointed out by Stuhmiller a long time ago when focusing on incompressible media, and more recently in the nuclear community. An annoying point is that this term only enlarges the domain of hyperbolicity ([22]), but in such a way that it cannot exactly recover the whole domain including all physically admissible states.

An alternative way to compute two-fluid models has emerged quite recently, which takes its grounds on the two-fluid two-pressure approach. One of the main advantages of the latter formalism is that it guarantees hyperbolicity for all admissible states (that is physical states with positive volume fractions, positive mass fractions and internal energies). However, many workers in the two-fluid community still seem to feel relunctant using these. The main objective here is to give a common framework which might unify both approaches, and meanwhile inherit of mathematical properties of the two-pressure closures.

The basic idea in this work is thus to take advantage of the structure of two-fluid two-pressure models to provide approximations on coarse meshes of standard two-fluid models. Actually, we show how to construct a simple numerical method which ensures that the maximum principle for the void fraction holds, and either leads to the prediction of the two-fluid two-pressure approach when using finite time relaxation, or to the standard two-fluid model when using zero relaxation time. Both rely on a control of the entropy inequality. We will also show, on the basis of a particular test case issuing from the literature, that the second algorithm hides deficiencies on coarse meshes which are nonetheless naturally retrieved when applying for hudge mesh refinement. This expected result is meaningful, and has already been pointed out some time ago in [7] in the framework of gas flow highly loaded with particles.

The whole algorithm is grounded on relaxation schemes. These have become quite popular over the past ten years, mainly because they enable to deal with complex EOS when computing gas dynamics with Euler equations (see [16] and [18] for instance). They are indeed useful to cope with nonlinear EOS, and enable to retrieve similar rates of convergence when compared with more standard exact or approximate Riemann solvers. The application to the frame of two-phase flows also seems very appealing. This has been recently investigated by F. Coquel and co-authors in order to cope with two-phase homogeneous models in industrial situations (see [3], [4], [5], [9], [11], $[12])$. The presentation of the paper is the following. We first provide the overall algorithm. We afterwards recall some properties of the two-fluid two-pressure model, and of the relaxation step. Schemes which are used to provide approximations within each step are then examined. The basic numerical example which is discussed in this paper is a simple Riemann problem issuing from the literature. We will focus on the standard two-fluid single pressure model. We briefly recall that this model leads to a blow-up of the computation when stabilizing drag effects are omitted, assuming the mesh is fine enough. We then consider the same initial conditions and account for drag effects with physical drag coefficients. For coarse or not too fine meshes, the numerical solution seems to be physically relevant ; however, the numerical approximation still blows up when one makes a -hudge- mesh refinement. Of course, results obtained with the two-pressure model do not suffer from this deficiency. 


\section{Two-fluid models and main algorithm}

We first introduce some classical notations. The subscript $k \in\{1,2\}$ will refer to the phase 1 and 2 respectively. Let $U_{k}, \rho_{k}$ and $P_{k}$ be the velocity, the density and the pressure within phase $k$. The volume fraction in each phase is denoted by $\alpha_{k} \in[0,1]$ with $\alpha_{1}+\alpha_{2}=1$. The partial masses are $m_{k}=\alpha_{k} \rho_{k}$, and $E_{k}=\rho_{k} e_{k}+\frac{\rho_{k} U_{k}^{2}}{2}$ stands for the total energy of phase $k$, where the internal energy is noted $e_{k}=e_{k}\left(P_{k}, \rho_{k}\right)$.

\subsection{The standard two-fluid model}

We first briefly recall that the standard two-fluid model (1) is a 6-equation model with two mass conservation equations, two momentum equations and two total energy equations.

$$
\left\{\begin{array}{l}
\frac{\partial m_{k}}{\partial t}+\frac{\partial m_{k} U_{k}}{\partial x}=0 \\
\frac{\partial\left(m_{k} U_{k}\right)}{\partial t}+\frac{\partial m_{k} U_{k}^{2}}{\partial x}+\alpha_{k} \frac{\partial P}{\partial x} \\
=T_{k} \\
\frac{\partial\left(\alpha_{k} E_{k}\right)}{\partial t}+\frac{\partial\left(\alpha_{k} U_{k}\left(E_{k}+P\right)\right)}{\partial x}+P \frac{\partial \alpha_{k}}{\partial t} \\
=T_{k} V^{i}
\end{array}\right.
$$

The interfacial momentum transfer $T_{k}$ in phase $k$ agregates the contributions of the drag force, which is:

$$
T_{k, 0}=(-1)^{k} K(W)\left(U_{1}-U_{2}\right)
$$

and the first order differential correction:

$$
T_{k, 1}=-\left(P-\pi^{i}\right) \frac{\partial \alpha_{k}}{\partial x}
$$

which vanishes when one assumes that $\pi_{i}=P$, and contributes to enlarge the hyperbolicity domain when $\pi_{i}-P=\beta\left(U_{2}-U_{1}\right)^{2}$ (see [21]). It is known from long that the eigenstructure of the convective part of this system can not be analytically exhibited. This lack of information obviously prevents us from building the exact solution of the Riemann problem, and also renders the construction of approximate Riemann solvers very tedious (see [21], [20]). But the most annoying point is that one also knows that some "elliptic" (with respect to time) areas remain, which results in a "blow-up" of the continuous solution, as illustrated in [19] (in other words, the initial boundary value problem becomes locally ill-posed). As a consequence, whatever the (assumed convergent) numerical method is, one expects that the approximate solution will also explode on sufficiently small grid sizes. We nonetheless underline that this strange pattern should not be confused with the preservation of the maximum principle for the volume fraction. Actually, regular solutions of the standard two-fluid model, agree with the maximum principle for $\alpha_{1}$, if the velocity fields (and their divergences) remain bounded over finite time intervals (see [6]). 


\subsection{The two-fluid two-pressure model}

This one may be written as:

$$
\left\{\begin{array}{l}
\frac{\partial \alpha_{1}}{\partial t}+V^{i} \frac{\partial \alpha_{1}}{\partial x}=\lambda\left(P_{1}-P_{2}\right) \\
\frac{\partial m_{k}}{\partial t}+\frac{\partial m_{k} U_{k}}{\partial x}=0 \\
\frac{\partial\left(m_{k} U_{k}\right)}{\partial t}+\frac{\partial\left(m_{k} U_{k}^{2}+\alpha_{k} P_{k}\right)}{\partial x}-P^{i} \frac{\partial \alpha_{k}}{\partial x}=T_{k} \\
\frac{\partial\left(\alpha_{k} E_{k}\right)}{\partial t}+\frac{\partial\left(\alpha_{k} U_{k}\left(\bar{E}_{k}+P_{k}\right)\right)}{\partial x}+P^{i} \frac{\partial \alpha_{k}}{\partial t}=T_{k} V^{i}
\end{array}\right.
$$

We will detail afterwards the specific forms of the interface velocity $V_{i}$ and interface pressure $P^{i}$. Setting in a formal way $\lambda=+\infty$ enables to retrieve the previous set associated with the standard two-fluid model.

The interfacial velocity and the interfacial pressure are modelled in a classical way (see [1], [2], [10], [13],[14], [15], [17] ):

$$
V_{i}=\mu U_{1}+(1-\mu) U_{2}
$$

where either $\mu(1-\mu)=0$ (see [2]), or $\mu=\frac{m_{1}}{m_{1}+m_{2}}$ (see [10]). Moreover:

$$
P^{i}=\frac{(1-\mu) a_{1} P_{1}+\mu a_{2} P_{2}}{(1-\mu) a_{1}+\mu a_{2}}
$$

where $a_{k}=\left(\frac{\partial e_{k}\left(P_{k}, \rho_{k}\right)}{\partial P_{k}}\right)^{-1} \frac{\partial \operatorname{Ln}\left(s_{k}\right)\left(P_{k}, \rho_{k}\right)}{\partial P_{k}}$.

\subsection{The basic algorithm}

Still using all above notations, we may now present the whole computational process between discrete time $t^{n}$ and $t^{n+1}$.

It consists in two steps :

- (i) an evolution step,

- (ii) and a relaxation step.

During the evolution step, we compute an approximate solution of the two-fluid two-pressure model, -the basics of which are recalled later on-, omitting the pressure relaxation terms. The initial condition in cell $i$ for this evolution step is $Z_{i}^{n}$, where $Z^{t}=\left(\alpha_{1}, m_{1}, m_{2}, m_{1} U_{1}, m_{2} U_{2}, \alpha_{1} E_{1}, \alpha_{2} E_{2}\right)$.

Thus the governing set of equations of the evolution step will be:

$$
\left\{\begin{array}{l}
\frac{\partial \alpha_{1}}{\partial t}+V^{i} \frac{\partial \alpha_{1}}{\partial x}=0 \\
\frac{\partial m_{k}}{\partial t}+\frac{\partial m_{k} U_{k}}{\partial x}=0 \\
\frac{\partial\left(m_{k} U_{k}\right)}{\partial t}+\frac{\partial m_{k} U_{k}^{2}}{\partial x_{2}}+\frac{\partial \alpha_{k} P_{k}}{\partial x}-P^{i} \frac{\partial \alpha_{k}}{\partial x}=T_{k} \\
\frac{\partial\left(\alpha_{k} E_{k}\right)}{\partial t}+\frac{\partial\left(\alpha_{k} U_{k}\left(E_{k}+P_{k}\right)\right)}{\partial x}+P^{i} \frac{\partial \alpha_{k}}{\partial t}=T_{k} V^{i}
\end{array}\right.
$$


Approximations at the end of the evolution process will be noted $\tilde{Z}$. Obviously, even if the local phasic pressures are in equilibrium in all cells at the beginning of the time step (that is: $\left.\left(P_{1}\right)_{i}^{n}=\left(P_{2}\right)_{i}^{n}\right)$, this will no longer hold at the end of the time step, which means that $\tilde{P}_{1} \neq \tilde{P}_{2}$.

The pressure-relaxation step will then correct the prediction in each cell, by computing approximations of the solution of the following set of ODE:

$$
\left\{\begin{array}{l}
\frac{\partial \alpha_{1}}{\partial t}=\lambda\left(P_{1}-P_{2}\right) \\
\frac{\partial m_{k}}{\partial t}=0 \\
\frac{\partial m_{k} U_{k}}{\partial t}=0 \\
\frac{\partial\left(\alpha_{k} E_{k}\right)}{\partial t}+P^{i} \frac{\partial \alpha_{k}}{\partial t}=0
\end{array}\right.
$$

Actually, both $m_{k}$ and $U_{k}$ remain unchanged during this process. In practice we shall set:

$$
\lambda=\alpha_{1} \alpha_{2} \theta^{-1} /\left(P_{1}+P_{2}\right)
$$

Hence, this in practice will update values of $\left(\alpha_{1}, P_{1}, P_{2}\right)$.

Now, two frames should be distinguished. In the first one, where one aims at computing true approximations of the two-fluid two-pressure model, the relaxation time $\theta$ associated with $\lambda$ should remain non zero. On the contrary, the standard two-fluid model requires instantaneous relaxation process $(\lambda=+\infty)$. The final time approximation is noted in any case $Z^{n+1}$.

We will focus herein on the computation of the standard two-fluid model, and thus will detail properties pertaining to the latter systems $(7-8)$ in the continuous and discrete frameworks. The reader is refered to [13] for details on the numerical procedures to cope with the relaxation process when $\lambda$ is finite. 


\section{Comments on both steps}

\subsection{Properties of the two-pressure model}

We very briefly recall herein some important properties of the two-fluid two-pressure model when the closures $(5-6)$ hold:

$$
\left\{\begin{array}{l}
\frac{\partial \alpha_{1}}{\partial t}+V^{i} \frac{\partial \alpha_{1}}{\partial x}=\lambda\left(P_{1}-P_{2}\right) \\
\frac{\partial m_{k}}{\partial t}+\frac{\partial m_{k} U_{k}}{\partial x}=0 \\
\frac{\partial\left(m_{k} U_{k}\right)}{\partial t}+\frac{\partial\left(m_{k} U_{k}^{2}+\alpha_{k} P_{k}\right)}{\partial x}-P^{i} \frac{\partial \alpha_{k}}{\partial x}=T_{k} \\
\frac{\partial\left(\alpha_{k} E_{k}\right)}{\partial t}+\frac{\partial\left(\alpha_{k} U_{k}\left(\bar{E}_{k}+P_{k}\right)\right)}{\partial x}+P^{i} \frac{\partial \alpha_{k}}{\partial t}=T_{k} V^{i}
\end{array}\right.
$$

The system (10) is hyperbolic. Unless some resonnance occurs, the set of eigenvectors spans $\mathbb{R}^{7}$. If we note $\rho_{k} c_{k}^{2}=\gamma_{k} P_{k}$, eigenvalues are real:

$$
\left\{\begin{array}{cll}
\lambda_{1}=V_{i} & \\
\lambda_{2}=U_{1}-c_{1}, & \lambda_{3}=U_{1}, & \lambda_{4}=U_{1}+c_{1} \\
\lambda_{5}=U_{2}-c_{2}, & \lambda_{6}=U_{2}, & \lambda_{7}=U_{2}+c_{2}
\end{array}\right.
$$

Fields 2,4,5,7 are genuinely non linear and the fields 1,3,6 are linearly degenerated (owing to (5)). Regular solutions of the latter system are such that $\alpha_{k}$ remain positive, assuming admissible inlet boundary conditions, and regular velocity fields. In addition, under the same assumptions, $m_{1}$ and $m_{2}$ remain positive. A similar property holds for $P_{k}$, for perfect gas laws and assuming that $\mu(1-\mu)=0$.

We need to introduce specific entropies $s_{k}$ in agreement with :

$$
\gamma_{k} P_{k} \frac{\partial s_{k}}{\partial P_{k}}+\rho_{k} \frac{\partial s_{k}}{\partial \rho_{k}}=0
$$

where:

$$
\rho_{k}\left(c_{k}\right)^{2}=\left(\frac{\partial e_{k}}{\partial P_{k}}\right)^{-1}\left(\frac{P_{k}}{\rho_{k}}-\rho_{k} \frac{\partial e_{k}}{\partial \rho_{k}}\right)
$$

A crucial point to note is that the following inequality holds for regular solutions:

$$
\frac{\partial \eta}{\partial t}+\frac{\partial F_{\eta}}{\partial x} \leq 0
$$

introducing:

$$
\eta=-m_{1} \operatorname{Ln}\left(s_{1}\right)-m_{2} \operatorname{Ln}\left(s_{2}\right)
$$

and:

$$
F_{\eta}=-m_{1} \operatorname{Ln}\left(s_{1}\right) U_{1}-m_{2} \operatorname{Ln}\left(s_{2}\right) U_{2}
$$

This is a keystone for the whole procedure. 


\subsection{Properties of the relaxation step}

We consider here the pressure relaxation step, that is:

$$
\left\{\begin{array}{l}
\frac{\partial \alpha_{1}}{\partial t}=\lambda\left(P_{1}-P_{2}\right) \\
\frac{\partial m_{k}}{\partial t}=0 \\
\frac{\partial m_{k} U_{k}}{\partial t}=0 \\
\frac{\partial \alpha_{k} E_{k}}{\partial t}-(-1)^{k} P^{i} \frac{\partial \alpha_{1}}{\partial t}=0
\end{array}\right.
$$

with an initial condition in the admissible range $\left(P_{k}>0, \alpha_{k}>0\right)$.

Throughout the pressure relaxation step, we note that:

$$
m_{k} \frac{\partial e_{k}}{\partial t}=-P^{i} \frac{\partial \alpha_{k}}{\partial t}
$$

and:

$$
\alpha_{k} \frac{\partial \rho_{k}}{\partial t}=-\rho_{k} \frac{\partial \alpha_{k}}{\partial t}
$$

Thus, we get:

$$
\frac{\partial s_{k}}{\partial t}=\left(\frac{\partial e_{k}}{\partial P_{k}}\right)^{-1} \frac{\partial s_{k}}{\partial P_{k}}\left(\frac{\partial e_{k}}{\partial t}-\frac{P_{k}}{\left(\rho_{k}\right)^{2}} \frac{\partial \rho_{k}}{\partial t}\right)
$$

or:

$$
\frac{\partial s_{k}}{\partial t}=\left(\frac{\partial e_{k}}{\partial P_{k}}\right)^{-1} \frac{\partial s_{k}}{\partial P_{k}}\left(\frac{P_{k}-P^{i}}{m_{k}}\right) \frac{\partial \alpha_{k}}{\partial t}
$$

Hence:

$$
\frac{m_{k}}{s_{k}} \frac{\partial s_{k}}{\partial t}=a_{k}\left(P_{k}-P^{i}\right) \frac{\partial \alpha_{k}}{\partial t}
$$

Since:

$$
\frac{\partial \eta}{\partial t}=-\sum_{k=1}^{2} \frac{m_{k}}{s_{k}} \frac{\partial s_{k}}{\partial t}
$$

we get :

$$
\frac{\partial \eta}{\partial t}=-\frac{a_{1} a_{2}}{(1-\mu) a_{1}+\mu a_{2}}\left(P_{1}-P_{2}\right) \frac{\partial \alpha_{1}}{\partial t}
$$

Thus :

$$
\frac{\partial \eta}{\partial t}=-\frac{a_{1} a_{2}}{(1-\mu) a_{1}+\mu a_{2}} \lambda\left(P_{1}-P_{2}\right)^{2}
$$

We may get some description of the miminum value obtained in $M_{0}$ :

$$
\frac{\partial \eta}{\partial t}=0 \quad<=>\quad P_{2}-P_{1}=0
$$

Even more, the second derivative around $M_{0}$ is:

$$
\left.\frac{\partial^{2} \eta}{\partial t^{2}}\right|_{M_{0}}=\frac{a_{1} a_{2}}{(1-\mu) a_{1}+\mu a_{2}}\left(\frac{\gamma_{1} P_{1}}{\alpha_{1}}+\frac{\gamma_{2} P_{2}}{\alpha_{2}}\right) X^{2}
$$


setting $X=\frac{\partial \alpha_{1}}{\partial t}$, and using the identity:

$$
\frac{\partial P_{k}}{\partial t}=\left(\frac{\partial e_{k}}{\partial P_{k}}\right)^{-1}\left(\frac{\partial e_{k}}{\partial t}-\left(\frac{\partial e_{k}}{\partial \rho_{k}}\right) \frac{\partial \rho_{k}}{\partial t}\right)
$$

Since $\gamma_{k} P_{k}=\rho_{k}\left(c_{k}\right)^{2}$ is positive, $\frac{\partial \eta}{\partial t^{2}}{ }_{M_{0}}$ is thus positive. This ensures that the instantaneous relaxation of pressure minimizes the entropy of the whole system. This result holds true for any EOS. 


\section{Schemes and main properties}

\subsection{A consistant relaxation method}

We only discuss herein the relaxation step when restricting to instantaneous relaxation. We need to introduce a notion of "consistancy condition on pressure" as follows. If we assume a perfect balance on initial values of pressures:

$$
\tilde{P}_{1}=\tilde{P}_{2}=\phi
$$

this should imply that:

$$
P^{n+1}=\phi
$$

and therefore: $\alpha_{k}^{n+1}=\tilde{\alpha_{k}}$. In other words, the relaxation step must be a "ghost step" in that particular case. We thus focus on the following scheme, which is exactly the one used in [8] :

$$
\left\{\begin{array}{l}
\left(P_{k}\right)_{i}^{n+1}=(P)_{i}^{n+1} \\
\left(m_{k}\right)_{i}^{n+1}=\left(\tilde{m}_{k}\right)_{i} \\
\left(m_{k} U_{k}\right)_{i}^{n+1}=\left(\tilde{m}_{k}\right)_{i}\left(\tilde{U}_{k}\right)_{i} \\
\left(\alpha_{k} E_{k}\right)_{i}^{n+1}-\left(\tilde{\alpha}_{k}\right)_{i}\left(\tilde{E}_{k}\right)_{i}+(P)_{i}^{n+1}\left(\left(\alpha_{k}\right)_{i}^{n+1}-\left(\tilde{\alpha_{k}}\right)_{i}\right)=0
\end{array}\right.
$$

The latter equation is equivalent to:

$$
\left(m_{k} e_{k}\right)_{i}^{n+1}-\left(\tilde{m_{k}}\right)_{i}\left(\tilde{e_{k}}\right)_{i}+(P)_{i}^{n+1}\left(\left(\alpha_{k}\right)_{i}^{n+1}-\left(\tilde{\alpha_{k}}\right)_{i}\right)=0
$$

owing to the first three mesh schemes.

If we moreover restrict to perfect gas EOS within each step, setting thus: $\left(\gamma_{k}-1\right) \rho_{k} e_{k}=P_{k}$, in agreement with condition $\gamma_{k}>1$, we immediatly get:

$$
\frac{\left(\alpha_{k} P_{k}\right)_{i}^{n+1}-\left(\tilde{\alpha}_{k} \tilde{P}_{k}\right)_{i}}{\gamma_{k}-1}+(P)_{i}^{n+1}\left(\left(\alpha_{k}\right)_{i}^{n+1}-\left(\tilde{\alpha_{k}}\right)_{i}\right)=0
$$

We deduce :

$$
P_{i}^{n+1}=\frac{\tilde{\alpha_{1 i}} \gamma_{2}\left(\tilde{P}_{1}\right)_{i}+\tilde{\alpha_{2 i}} \gamma_{1}\left(\tilde{P}_{2}\right)_{i}}{\tilde{\alpha_{1 i}} \gamma_{2}+\tilde{\alpha_{2 i}} \gamma_{1}}
$$

and subsequently $\left(P_{k}\right)_{i}^{n+1}=P_{i}^{n+1}$ (for $\left.k=1,2\right)$, but also:

$$
\left(\alpha_{k}\right)_{i}^{n+1}=\left(\tilde{\alpha}_{k}\right)_{i}\left(\frac{\gamma_{k}-1}{\gamma_{k}}+\frac{\left(\tilde{P}_{k}\right)_{i}}{\gamma_{k} P_{i}^{n+1}}\right)
$$

Property 1:

Both $P_{i}^{n+1}$ and $\left(\alpha_{1}\right)_{i}^{n+1}\left(1-\alpha_{1}\right)_{i}^{n+1}$ remain positive through the relaxation step, assuming relevant initial data. 


\subsection{Basic schemes used to compute the evolution step}

The numerical method used to compute approximate solutions of the convection step is exactly the one described in [13]. It is thus not recalled in this paper. It is a Finite volume method, where the numerical convective fluxes at each cell interface rely either on Rusanov scheme, the exact Godunov scheme or on the approximate Godunov scheme VFRoe-ncv (see [12]). The time step is chosen to agree with the standard CFL condition: $C F L=0.5$.

Property 2:

2.1- The Rusanov scheme enables to maintain positive values of the void fraction and partial masses, assuming standard CFL condition.

2.2- The exact Godunov scheme preserves the positivity of expected positive components.

Obviously, if one aims at computing the standard two-fluid model, one needs to initialize first the "mean pressure" $(P)_{i}^{0}$, and then to define the cell phasic pressures as follows: $\left(P_{1}\right)_{i}^{0}=(P)_{i}^{0}$ and $\left(P_{2}\right)_{i}^{0}=(P)_{i}^{0}$, for any cell with subscript $i$. The whole procedure will then ensure that a pressure equilibrium is achieved at the end of each time step, owing to the relaxation procedure. 


\section{$5 \quad$ Numerical results}

We note that we use afterwards the Baer-Nunziato closure, which means that $\mu=0$, which turns to $V_{i}=U_{2}$, and $P^{i}=P_{1}$.

\subsection{The Riemann problem}

We consider initial conditions issuing from [8]. Thus, we start the computation with :

$$
\begin{gathered}
U_{1}(x, t=0)=U_{2}(x, t=0)=0 \\
\alpha_{1}(x, t=0)=0.25 \quad P(x, t=0)=2.10^{7} \\
h_{1}(x, t=0)=3.09310^{6} \quad h_{2}(x, t=0)=1.3510^{6}
\end{gathered}
$$

for $x<0.5$, and :

$$
\begin{gathered}
U_{1}(x, t=0)=U_{2}(x, t=0)=0 \\
\alpha_{1}(x, t=0)=0.25 \quad P(x, t=0)=1.510^{7} \\
h_{1}(x, t=0)=3.09310^{6} \quad h_{2}(x, t=0)=2.3510^{6}
\end{gathered}
$$

for $x>0.5$. A coarse mesh contains 50 cells, a medium mesh contains 1000 cells, and the finer mesh contains 250000 cells. The straight line corresponds to the computation where drag effects have been omitted, squares denote approximations where the drag coefficient is computed with a bubble particle mean diameter $d=1.10^{-3}$, and circles represent solutions for $d=1.10^{-6}$.

For each computation, we plot the modulus of non zero imaginary part of eigenvalues, if meaningful. The physical time for simulation is the same in each case.

\subsection{Omitting drag effects}

Figures 1, 2, 3 and 4 show the void fraction, the mean pressure and velocities. Figure 5 shows that some complex eigenvalues arise in the computation, which are obviously correlated with the relative velocity $U_{r}$, but the coarse mesh with 50 cells provides a smooth solution. The solution nonetheless shows a strange behaviour on the void fraction, mean pressure, velocity and the relative velocity $U_{2}-U_{1}$ profiles when using a mesh with 1000 cells (see figures $6,7,8,9,10$ ). The solution explodes when using a mesh with 5000 cells.

\subsection{Accounting for drag effects}

When accounting for drag effects, the order of magnitude of the relative velocity is clearly reduced. Focusing on the mesh with 1000 cells, it occurs that the pressure profile is much smoother (see figures 7 ). This holds true for velocities too.

Nonetheless, we note that the imaginary part of eigenvalues is non zero when $d=10^{-3}$ (see figure 10). If one turns then to the same particle size on a much finer mesh with 250000 cells, it clearly 


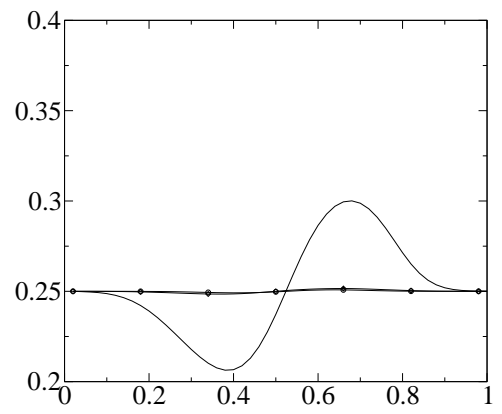

Figure 1: Riemann problem 1: void fraction. Straight line (no drag), squares $\left(d=1.10^{-3}\right)$, $\operatorname{circles}\left(d=1.10^{-6}\right)-50$ cells

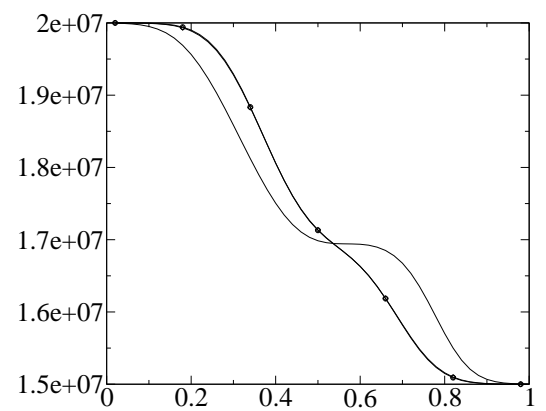

Figure 2: Riemann problem 1: mean pressure P. Straight line (no drag), squares $\left(d=1.10^{-3}\right)$, $\operatorname{circles}\left(d=1.10^{-6}\right)-50$ cells

arises that the void fraction is once again much polluted around the location $x=0.52$ (see figure 11). An amazing point is that the pressure profile still seems to be regular around this location on this particular mesh (see figure 12). Velocities are plotted on figures 13 and 14 . Figure 16 displays the correlation between the void fraction and the relative velocity around $x=0.52$.

Actually, one retrieves a blow up of the code on a mesh with $10^{6}$ cells. 


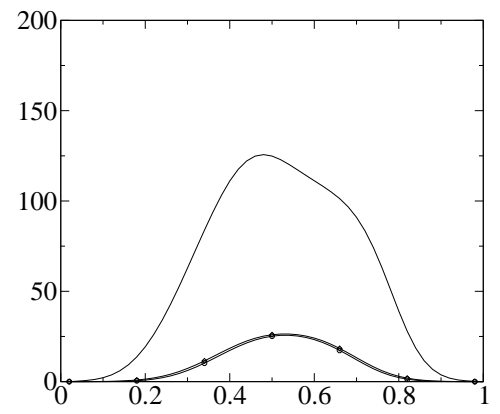

Figure 3: Riemann problem 1: velocity $U_{1}$. Straight line (no drag), squares $\left(d=1.10^{-3}\right)$, $\operatorname{circles}\left(d=1.10^{-6}\right)-50$ cells

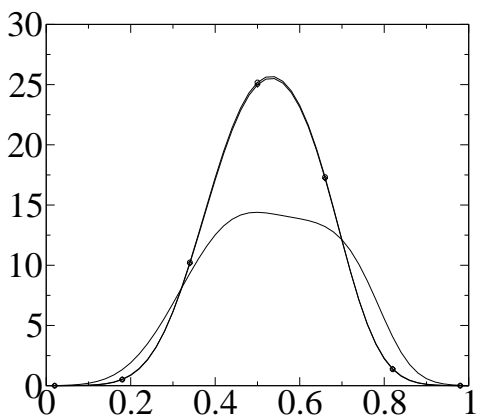

Figure 4: Riemann problem 1: velocity $U_{2}$. Straight line (no drag), squares $\left(d=1.10^{-3}\right)$, $\operatorname{circles}\left(d=1.10^{-6}\right)-50$ cells

\section{Conclusion}

We have a twofold conclusion:

(i) The numerical method which has been proposed herein, which combines the use of the two-fluid two-pressure approach and the relaxation procedure, enables to cope with both the two-fluid twopressure model and the standard two-fluid model, without specific restriction on the time stepping ;

(ii) The overall algorithm is stable, and fortunately does not hide potential deficiencies of continuous models regarding hyperbolicity.

It may thus be seen as a possible tool to compute both well-posed initial-value problems on any mesh, and possibly locally ill-posed models on "coarse meshes". 


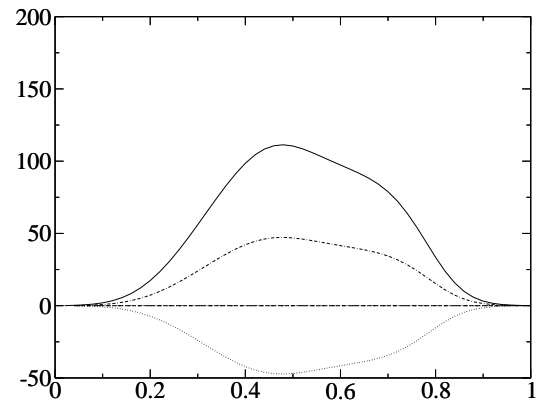

Figure 5: Riemann problem 1: velocity $U_{r}$ and imaginary part of eigenvalues. (no drag) - 50 cells

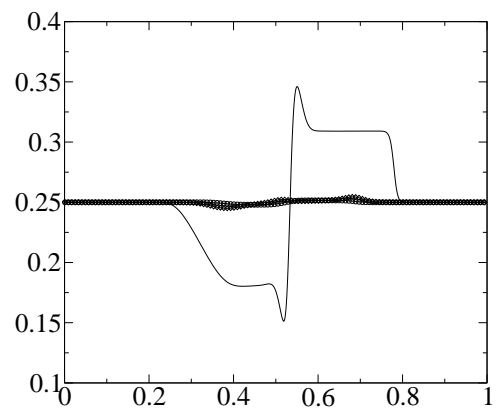

Figure 6: Riemann problem 1: void fraction. Straight line (no drag), squares $\left(d=1.10^{-3}\right)$, $\operatorname{circles}\left(d=1.10^{-6}\right)-1000$ cells

\section{Acknowledgements}

This work was supported by EDF (Eléctricité de France) in the framework of the Neptune project. Computational facilities were provided by EDF. The second author receives financial support under CIFRE/EDF contract. 


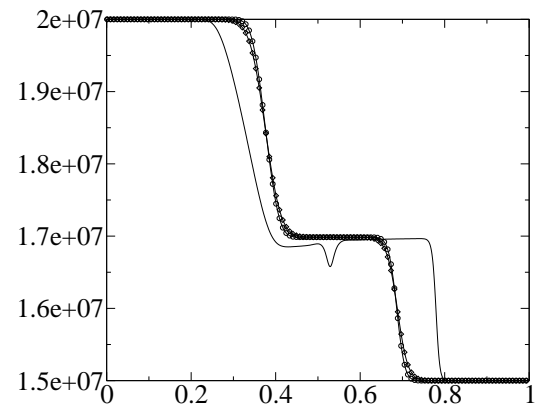

Figure 7: Riemann problem 1: mean pressure P. Straight line (no drag), squares $\left(d=1.10^{-3}\right)$, $\operatorname{circles}\left(d=1.10^{-6}\right)-1000$ cells

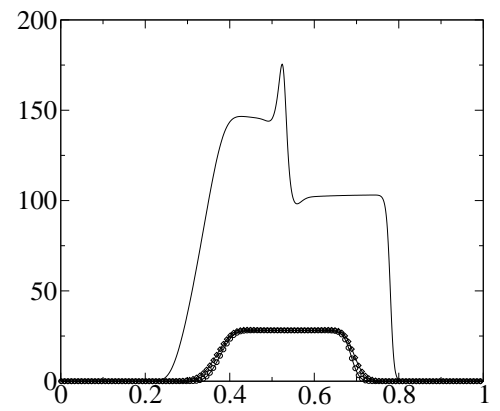

Figure 8: Riemann problem 1: velocity $U_{1}$. Straight line (no drag), squares $\left(d=1.10^{-3}\right)$, $\operatorname{circles}\left(d=1.10^{-6}\right)-1000$ cells

\section{References}

[1] N. Andrianov and G. Warnecke, The riemann problem for the baer nunziato two-phase flow model, J. of Comp. Physics., 2004, vol. 195, pp. 434-464.

[2] M.R. Baer and J.W. Nunziato, A two phase mixture theory for the deflagration to detonation (ddt) transition in reactive granular materials, Int. J. for Multiphase Flow, 1986, vol. 12-6, pp. 861-889.

[3] P. Bagnerini, F. Coquel, E. Godlewski, P. Marmignon et al., A void fraction relaxation principle for averaged two phase flow models, preprint laboratoire Dieudonné, 2002.

[4] M. Baudin, C. Berthon, F. Coquel, R. Masson et al., A relaxation method for twophase flow models with hydrodynamic closure law, Numerische Mathematik, 2005, vol. 99, pp. $411-440$.

[5] F. Caro, F. Coquel, D. Jamet and S. Kokh, Dinmod : a diffuse interface model for two-phase flows modelling, internal CEA report DEN/DM2S/SFME/LETR, 2004.

[6] S. Clerc, T. Gallouët, J.M. Hérard and N. Seguin, Partial review of positivity constraints in some two phase flow models, AIAA paper 2002-3185, 2002. 


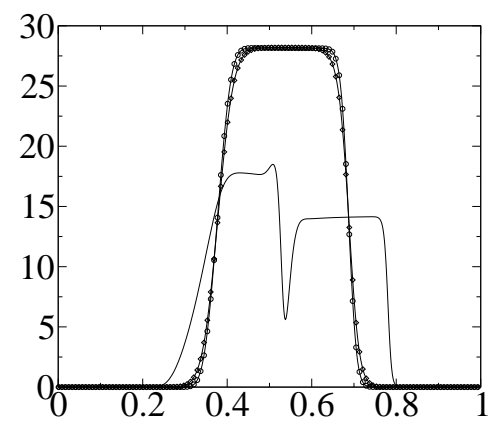

Figure 9: Riemann problem 1: velocity $U_{2}$. Straight line (no drag), squares $\left(d=1.10^{-3}\right)$, $\operatorname{circles}\left(d=1.10^{-6}\right)-1000$ cells

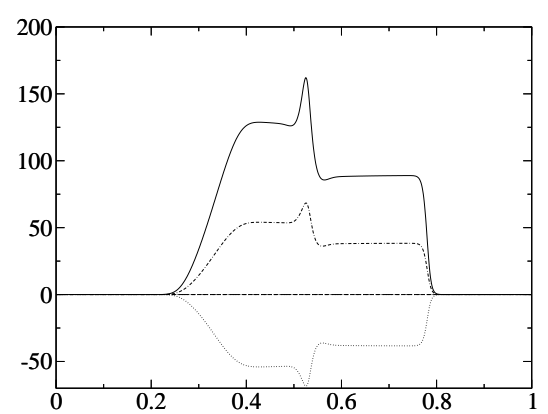

Figure 10: Riemann problem 1: velocity $U_{r}$ and imaginary part of eigenvalues. (no drag) - 1000 cells

[7] L. Combe and J.M. HÉrard, Finite volume algorithm to compute dense compressible gassolid flows, AIAA journal, 1999, vol. 37, pp. 337-345.

[8] F. Coquel, K. El Amine, E. Godlewski, B. Perthame et al., A numerical method using upwind schemes for the resolution of two phase flows, J. Comp. Phys., 1997, vol. 136, pp. $272-288$.

[9] F. Coquel And S. Cordier, MATAPLI 62, 2000, pp. 27-58.

[10] F. Coquel, T. GallouËt, J.M. Hérard and N. Seguin, Closure laws for a two-fluid two-pressure model, C. R. Acad. Sci. Paris, 2002, vol. I-334, pp. 927-932.

[11] F. Coquel and B. Perthame, Relaxation of energy and approximate Riemann solvers for general pressure laws in fluid dynamics equations, SIAM J. Numer. Anal., 1998, vol. 35-6, pp. 2223-2249. In Memory of Ami Harten.

[12] T. Gallouët, J.M. Hérard and N. Seguin, Some recent Finite Volume schemes to compute Euler equations using real gas EOS, Int. J. for Num. Meth. in Fluids, 2002, vol. 39, pp. 1073-1138.

[13] _ Numerical modelling of two-phase flows using the two-fluid two-pressure approach, Math. Mod. Meth. in Appl. Sci., 2004, vol. 14-5, pp. 663-700. 


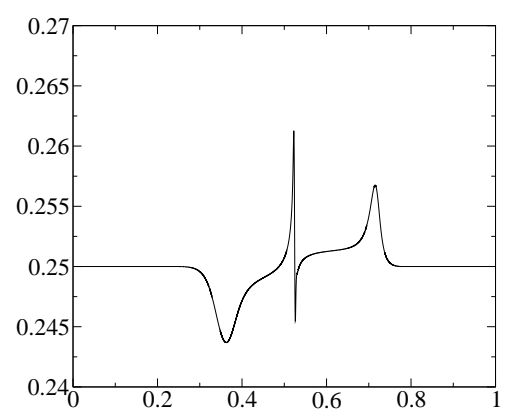

Figure 11: Riemann problem 1: void fraction. $\left(d=1 \cdot 10^{-3}\right)-250000$ cells

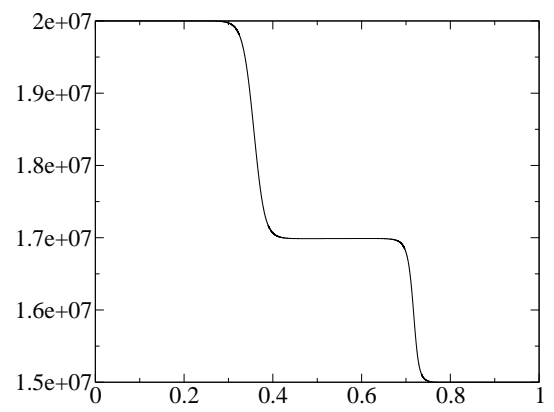

Figure 12: Riemann problem 1: mean pressure. $\left(d=1.10^{-3}\right)-250000$ cells

[14] S. Gavrilyuk and R. Saurel, Mathematical and numerical modelling of two phase compressible flows with inertia, J. Comp. Phys., 2002, vol. 175, pp. 326-360.

[15] J.M. HÉRARD, Numerical modelling of turbulent two phase flows using the two-fluid approach, AIAA paper 2003-4113, 2003.

[16] S. Jin AND Z. XIN, The relaxation schemes for systems of conservation laws in arbitrary space dimensions, Communications in Pure and Applied Mathematics, 1995, vol. 48, pp. 235-276.

[17] A.K. Kapila, S.F. Son, J.B. Bdzil, R. Menikoff et al., Two-phase modelling of ddt : structure of the velocity relaxation zone, Physics of Fluids, 1997, vol. 9, pp. 3885-3897.

[18] R.J. LeVeque and M. Pelanti, A class of approximate riemann solvers and their relation to relaxation schemes, J. Comp. Phys., 2001, vol. 172, pp. 572-591.

[19] J.M. Rovarch, A multidimensional Finite Volume method with physical variables implicitation - application to two-phase flows, in proceedings of FVCA III, Hermes Penton Science editor, 2002, pp. 647-654.

[20] I. TiselJ and S. Petelin, Modelling of two-phase flow with second accurate scheme, $J$. Comp. Phys., 1997, vol. 136, pp. 503-521.

[21] I. Toumi And A. Kumbaro, An approximate linearized Riemann solver of a two-fluid model, J. Comp. Phys., 1996, vol. 124, pp. 286-300. 


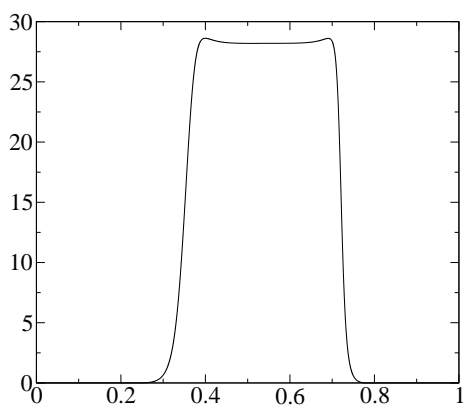

Figure 13: Riemann problem 1: $U_{1} \cdot\left(d=1.10^{-3}\right)-250000$ cells

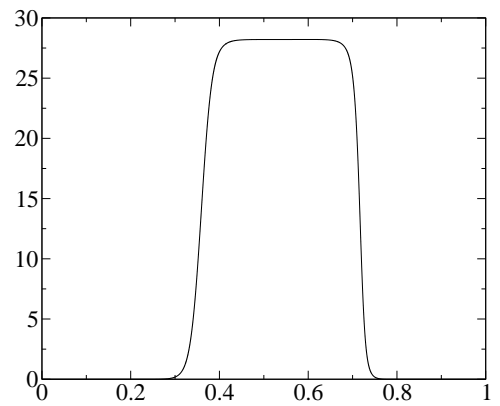

Figure 14: Riemann problem 1: $U_{2} \cdot\left(d=1.10^{-3}\right)-250000$ cells

[22] M. Valette and S. Jayanti, Annular dispersed flow calculations with a two-phase three field model, European Two phase Flow Group Meeting, Norway, 2003. 


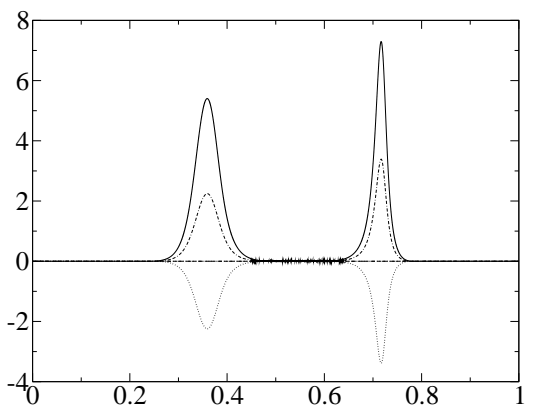

Figure 15: Riemann problem 1: relative velocity $U_{r}$ and imaginary part of eigenvalues. $\left(d=1.10^{-3}\right)$ - 250000 cells

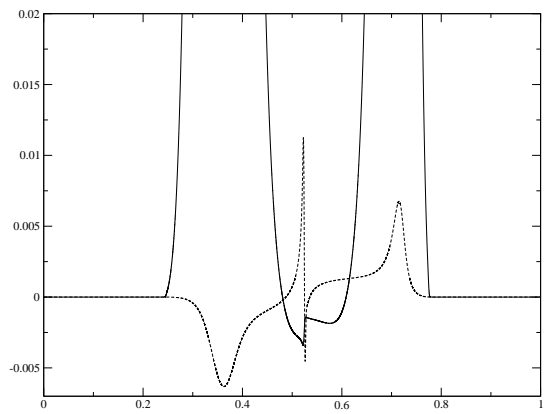

Figure 16: Riemann problem 1: zooming the relative velocity $U_{r}$ and the void fraction. $\left(d=1.10^{-3}\right)$ - 250000 cells 